\title{
Are Chronic Pain Patients with Dementia Being Undermedicated?
}

This article was published in the following Dove Press journal:

Journal of Pain Research

\author{
Wilco P Achterberg (D) \\ Ane Erdal $\mathbb{1 D}^{2}$ \\ Bettina S Husebo $\mathbb{D}^{2}$ \\ Miriam Kunz ${ }^{3}$ \\ Stefan Lautenbacher (iD ${ }^{4}$ \\ 'Department of Public Health and \\ Primary Care, Leiden University Medical \\ Center, RC Leiden, 2300, the \\ Netherlands; ${ }^{2}$ Department of Global \\ Public Health and Primary Care, \\ University of Bergen, Bergen, 5020, \\ Norway; ${ }^{3}$ Department of Medical \\ Psychology, University of Augsburg, \\ Augsburg, 86I56, Germany; ${ }^{4}$ Department \\ of Physical Psychology, University of \\ Bamberg, Bamberg, Germany
}

\begin{abstract}
In dementia, neuropathological changes alter the perception and expression of pain. For clinicians and family members, this knowledge gap leads to difficulties in recognizing and assessing chronic pain, which may consequently result in persons with dementia receiving lower levels of pain medication compared to those without cognitive impairment. Although this situation seems to have improved in recent years, considerable geographical variation persists. Over the last decade, opioid use has received global attention as a result of overuse and the risk of addiction, while the literature on older persons with dementia actually suggests undertreatment. This review stresses the importance of reliable assessment and the regular evaluation and monitoring of symptoms in persons with dementia. Based on current evidence, we concluded that chronic pain is still undertreated in dementia.
\end{abstract}

Keywords: pain, dementia, assessment, analgesic treatment

\section{Introduction}

In 1901, Auguste Deter, a 51-year-old woman committed to the Frankfurt Asylum, was showing short-term memory problems and unusual behavioral symptoms. A medical doctor at the asylum, Alois Alzheimer, was intrigued by her case and closely followed the patient, eventually studying post-mortem brain autopsy material. ${ }^{1}$ The clinical picture, combined with amyloid plaques and neurofibrillary tangles in the brain, was the first description of what we now refer to as 'Alzheimer's disease', one of the many forms of dementia. ${ }^{2}$ Unfortunately, it is not known whether Auguste also had pain, and if so, whether this was noticed by Dr Alzheimer or other caregivers. If something had been noticed, would it have been recognized as pain? And if recognized, would it have been treated?

In this review, we discuss the problems surrounding the perception, expression, recognition and treatment of pain in persons with dementia, with the ultimate goal of answering the question "Are chronic pain patients with dementia currently undermedicated?".

\section{Methodology}

For the purposes of this narrative review, we carried out a literature search in PubMed using the search terms pain, dementia, management, medication and undertreatment. As an abundance of review papers have appeared over the last 15 years, we decided to specifically focus on studies published between 2015 and August 2020.
Department of Public Health and Primary Care, Leiden University Medical Center, Hippocratespad 2I, Postzone V0-P, Room V6-76, PO Box 9600, RC Leiden, 2300 the Netherlands

Tel +3 I 7I 52684 I 2/8427;

$+31623668980$

Email w.p.achterberg@lumc.nl 
However, we should provide some background on why the undertreatment of pain in dementia is an important topic. We will first describe the epidemiology and neuropathology of dementia, followed by the changes in pain perception and expression in dementia that have consequences for assessment.

\section{Epidemiology of Dementia}

Dementia as a syndrome involves a range of functional impairments that affect memory and executive functions (amongst other cognitive functions), motor and sensory aspects, and communicative, behavioral and psychological functions. ${ }^{3}$ The disease trajectory is very heterogeneous and thus unpredictable, but diagnosis to death usually takes up to 10 years, depending on personal and diseaserelated factors. ${ }^{4}$ Many diseases characterized by specific neuropathological changes can lead to the syndrome of dementia, of which Alzheimer's disease is the most common. $^{5}$ Although Alzheimer's was described over 100 years ago and the formation of tangles and plaques was already recognized in the Auguste Deter case, disease onset and the complete etiological pathway is still not fully understood. Therefore, a cure is also currently beyond reach.

Vascular dementia, Lewy-body dementia and frontotemporal dementia are other more or less prevalent diseases that cause dementia, although there is substantial heterogeneity in causes worldwide. ${ }^{3}$ Vascular dementia is typified by white matter damage as a consequence of vascular pathology, such as long-term hypertension. Alzheimer's dementia and vascular dementia are so common that the mixed presence of these two forms of dementia may, in fact, be the most prevalent clinical picture in older individuals. ${ }^{3}$ Other neurodegenerative diseases, such as Parkinson's disease and Huntington's disease, may in the later stages also lead to a form of dementia. ${ }^{6,7}$

\section{Changes in Pain Perception in Dementia}

The neuropathological changes in various types of dementia disrupt elaborate neural pain networks, ${ }^{8}$ such as the lateral and medial pain networks involved in pain perception. The lateral network is the oldest evolutionary pain system and indeed the most 'basic', as it mediates the sensory-discriminative aspects of pain, such as localization, intensity and quality of pain. It is located in the primary somatosensory cortex and the (lateral) thalamus, amongst other sites. Cognitive, evaluative and motivational-affective aspects of pain are thought to be mediated via the medial network, which is comprised of the prefrontal cortex and several subcortical structures such as the amygdala, hippocampus and thalamus. ${ }^{9}$ A third pain network comprised of various systems including the striatum is believed to be the rostral, or limbic pain system, which mediates behavioral reactions to pain. ${ }^{10}$

In Alzheimer's dementia, the medial pain network shows greater neuropathology and more damage than the lateral network. At one time, researchers assumed that Alzheimer's patients might have an unchanged pain threshold but with a higher pain tolerance due to the damaged cognitive-evaluative functions. Although early experimental studies seemed to confirm this theory, later studies of EEG responses to pain, pain reflexes, studies of facial responses to noxious stimuli and fMRI studies during pain stimuli all showed that if there is a change, it is probably towards a lower pain tolerance and the level of suffering is likely to be higher. ${ }^{11,12}$

As a key player in the rostral or limbic pain system, and specifically associated with pain behavior, the striatum is affected by Alzheimer's disease. However, studies have produced conflicting results, showing either a lower or higher activation of the striatum in response to pain, depending on the stage of dementia. It is generally believed that activation of the striatum in response to pain is stronger in mild to moderate Alzheimer's disease, but is normal or even absent in later stages. This is in line with clinical observations and some clinical studies which suggest that behavioral reactions to pain are blunted in the later stages of Alzheimer's disease. ${ }^{11}$

\section{Changes in Pain Expression in Dementia}

During the course of dementia, the ability to recognize, evaluate and verbally express one's own pain gradually decreases. ${ }^{11,12}$ Thus, whereas self-reported pain is viewed as the most valid channel of pain expression in cognitively healthy individuals, this channel loses its validity in dementia and other non-verbal channels of pain expression become more important. The best-studied alternative has been the facial expression of pain, ${ }^{13,14}$ and several studies have used experimental designs that involved pain induction based on mechanical pressure, electrical stimulation, flu injection, venipuncture or the exacerbation of chronic musculoskeletal pain during physical exercise. During the 
experimental induction of pain, facial expressions were videotaped and later analyzed by specially trained raters using the Facial Action Coding System (FACS). ${ }^{15}$ FACS is an anatomically based method designed to objectively describe detailed facial muscle movements and is widely seen as the gold standard when assessing facial responses to pain. ${ }^{16}$ Four facial movements have been repeatedly observed in the context of pain, consisting of a tightening of the muscles surrounding the eyes, furrowed brows, raising the upper lip/nose wrinkling and an opened mouth. ${ }^{16}$ It is important to realize that these facial movements are not typically displayed together when somebody is in pain, as individuals usually show different mixtures of each facial movement. ${ }^{17,18}$ The most frequent response to pain is a tightening of the muscles around the eyes, paired with one or two other pain-indicative facial movements.

\section{Impact of Dementia on Facial Responses}

Findings on the impact of dementia on facial responses to pain are surprisingly homogeneous, despite the use of different pain induction methods and the study of mixed samples of patients suffering from dementia. It has been consistently found that as pain intensity increases, facial responses intensify in patients with dementia. ${ }^{13-18}$ This clearly indicates that facial expressions in patients with dementia reliably convey pain intensity. Furthermore, the facial responses to pain in patients with dementia are the same as those in individuals without dementia. ${ }^{13,15,16}$ In terms of the strength and extent of facial responses to pain in patients with dementia, it was found that patients actually show increased facial displays of pain compared to the cognitively unimpaired. ${ }^{13,14}$ The increased facial expression of pain might suggest an intensified processing of noxious stimuli and/or a loss of inhibitory mechanisms that govern the degree to which we express pain via facial expressions. In summary, while individuals with dementia and cognitively healthy individuals express pain using the same types of facial muscle movements, individuals with dementia show more vigorous and intense responses.

\section{New Developments in Recognition/ Assessment in Dementia}

An essential requirement for acceptable pain management is a well-performed pain assessment that covers the different aspects of pain (eg intensity, location, affect, cognition, behavior, social accompaniments). However, communication difficulties and cognitive impairment in patients with dementia mean that it is not possible to focus on all of these aspects. Thus, limited and one-sided pain assessment (focusing only on the presence and intensity of pain) cannot be avoided in individuals with dementia. Below we discuss several of the best pain assessment practices for persons with dementia.

\section{Self-Report}

Despite early declines in cognitive and language capacities, self-report should still be used, or at least attempted, in patients in the earlier phases of dementia (mild to moderate). ${ }^{19}$ It is advisable to use less cognitively challenging scales such as simple numerical scales (eg numerical rating scales (NRS) ranging from " 0 " (no pain) to " 10 " (extremely strong pain)) or verbal category scales (eg a 5-point Likert scale: "no pain", "mild pain", "moderate pain", "strong pain", "very strong pain"). As dementia progresses, simple questions that only require categorical answers such as 'yes/no' should be used.

\section{Observational Scales}

As cognitive and communication skills weaken due to the progression of dementia, self-report questions should be accompanied by assessment tools based on clinical observation. ${ }^{20}$ In the later stages of dementia, observational pain assessment takes a leading role in pain assessment. These scales mainly use facial expressions and vocal responses to pain, in addition to body posture and movement. ${ }^{20,21}$ One of the difficulties with these observational instruments, of which there are more than ten, lies in psychometric properties that are often poor or untested. ${ }^{20}$ These include inter- and intra-rater reliability, validity and sensitivity to change. The best available observational pain instruments, although not perfect, are PAINAD, ${ }^{22,23}$ PACSLAC, ${ }^{24}$ and DOLOPLUS-2. ${ }^{25,26}$ Another issue related to poor pain management in persons with dementia is a poor implementation in practice. That is unfortunate because the comprehensive and systematic implementation of these tools improves clinical practice. The focus should, therefore, not only be on psychometric testing but should also certainly include informing and educating the main end-user group, the long-term care and geriatric nurses. This group should be tasked with applying these tools but can also help further improve the usability of these tools. ${ }^{11}$

The last 5 years have seen two initiatives designed to tackle the problem of imperfectly developed observational tools. Both approaches used a meta-tool concept, which involves selecting the best items from existing observer 
rating scales. A USA-based group developed the 'Pain Intensity Measure for Persons with Dementia' (available in English), ${ }^{27}$ while a European group developed the 'Pain Assessment in Impaired Cognition' (PAIC-15; available in English and 8 other languages, including Chinese and Japanese). ${ }^{28,29}$ Both instruments are very promising, and a number of studies of their properties and clinical usefulness are currently underway.

\section{Automatic Pain Recognition}

When communication becomes difficult and behavioural observation complicated, one might consider more technical solutions to recognize and assess signals of pain. However, despite a considerable promise in noncommunicative patients, the automation of pain recognition faces many challenges. ${ }^{30}$ The most promising of these automated pain tools involves the monitoring of facial expressions, sometimes together with other biological cues such as blood pressure, heart and cerebral function (ECG and EEG), and occasionally, actigraphy. ${ }^{31}$ Currently, machine learning algorithms are often developed under laboratory conditions in terms of surroundings, are designed to assess bedridden patients and often lack reallife emotional expressions. In addition, this form of computer-driven pain diagnostic has mainly been developed in a young population, without taking account of the specifics of older populations. This may lead to the so-called 'wrinkle' bias, which is a lack of validation for older faces. ${ }^{30}$ Together, these issues mean that the use of these tools in clinical geriatric practice remains in the distant future and progress in this promising field will only be possible based on collaboration between the relevant clinicians (nurses, physicians, psychologists) and computer, engineering and artificial intelligence scientists.

\section{New Developments in Pain Management: Is Undertreatment an Issue?}

In an earlier review in 2013, we described several studies, encompassing a number of care settings, that reported undertreatment of patients with dementia compared to persons without cognitive impairment. ${ }^{11}$ Four studies provided particularly clear evidence of a lower use of analgesics in persons with dementia compared to those without dementia. ${ }^{32-35}$ However, one study showed almost no difference, ${ }^{31}$ and two reported a higher use of pain medication in persons with dementia. ${ }^{32,33}$ There were almost no data on the use of non-pharmacological management, but the conclusion was that, in general, undertreatment of persons with dementia is very common. Undertreatment is thought to be driven by complications surrounding the recognition and assessment of pain, together with reservations concerning polypharmacy and the side effects of medication. We and others concluded that poor pain management of persons with dementia places a substantial burden on their quality of life. ${ }^{36}$ However, in the last few years, the rapidly growing use of opioids has received considerable attention - prompting us to revisit opioid treatment of persons with dementia.

\section{Treatment with Opioids}

Opioid analgesics are commonly used for acute and chronic non-cancer pain in people with dementia, although their efficacy and safety, particularly during long-term use, is supported by little evidence or documentation. ${ }^{20,37}$ Because clinical evidence is lacking, no dementiaspecific treatment guidelines exist for moderate to severe acute or chronic pain. ${ }^{20}$ In 2019, the International Association for the Study of Pain published a fact sheet on 'Pain Management in Dementia' as part of the 'Global Year Against Pain in the Most Vulnerable', stating that while strong opioids may be used cautiously, weak opioids (eg codeine, tramadol) should be avoided in those with dementia due to high interindividual variation in treatment effect due to metabolic activation, little evidence for effectiveness, and a high risk of adverse effects and drug interactions (https://www.iasp-pain.org/GlobalYear/ MostVulnerable). In practice, prescription rates vary widely, both between and within countries and regions.

\section{Discrepancies Between Nations}

Several studies published in the last 5 years illustrate the major inconsistencies in prescribing practices and pain management guidelines between nations. In Poland, less than $3 \%$ of a sample of nursing home patients with mild cognitive impairment or dementia (MMSE $\leq 23$ ) were treated with an opioid, with the majority receiving only the weak opioid tramadol. ${ }^{37}$ The same study found that $90 \%$ of patients with severe cognitive impairment received no analgesic treatment, compared to $76 \%$ of those with no cognitive impairment $(\mathrm{p}<0.01) .{ }^{38}$ In 2012 , an Italian study also showed low opioid prescription in a sample of hospitalized geriatric patients, $6.6 \%$ of whom received an opioid at discharge. The corresponding rate in 116 patients diagnosed with dementia was only $2.6 \%\left(\chi^{2}\right.$ test, $\left.\mathrm{p}=0.126\right){ }^{39}$ 
In contrast, a recent study from Austria reported a high use of opioids among 425 nursing home patients from 12 nursing homes, with $50.2 \%$ receiving permanently scheduled opioid prescriptions, with no significant differences between people with no/slight and moderate-to-severe cognitive impairment. ${ }^{38}$

\section{USA}

A recent study from the USA, by Mehta et al (2020), investigated a cohort of 734,739 long-term nursing home residents (from 2011 to 2017) and found that advanced dementia was associated with a less opioid prescription. As analyses were controlled for the presence of pain, this finding potentially reflects suboptimal pain treatment. ${ }^{40}$ Between 2011 and 2017, there was a 7.5\% relative reduction in any opioid use among people with no dementia (from $48.2 \%$ to $44.6 \%$ ), a $14.1 \%$ relative reduction among those with mild dementia (from $38.9 \%$ to $33.4 \%$ ), a $13.1 \%$ relative reduction among people with moderate dementia (from $28.3 \%$ to $24.6 \%$ ), and a $10.8 \%$ relative reduction among people with severe dementia (from $24.1 \%$ to $21.5 \%){ }^{40}$ These results are supported by Hunnicutt et al (2018) who investigated opioid use in a sample of 315,949 Medicare beneficiaries with long-term nursing home stays in 2012 and found that among those with persistent pain, $38.7 \%$ of people with no/mild cognitive impairment used opioids long-term; in moderate and severe dementia the corresponding rates were significantly lower at $34.1 \%$ and $31.1 \%$, respectively. ${ }^{41}$ Shen et al (2018) investigated a sample of 19,347 elderly community-dwelling Medicare beneficiaries (2006 to 2013) and found that people with Alzheimer's disease and related dementias (ADRD) were significantly less likely to receive opioids than those without ADRD (32.8\% vs 33.4\%), when analyses were controlled for general health status (OR 0.82, $95 \%$ CI $0.71-0.94){ }^{42}$

\section{The Opioid Epidemic in Dementia}

While the overall use of opioids in community-dwelling elderly people increased from 2006 to $2013,{ }^{42}$ declining trends for opioid prescribing were reported in nursing home patients with and without dementia from 2011 to $2017,{ }^{40}$ potentially reflecting a change in prescribing practice as a result of increased attention for the prevention of opioid addiction and opioid overdose deaths associated with the opioid epidemic in the USA. Despite an apparent shift in prescribing practice, the likelihood of receiving opioid treatment remains significantly lower in people with dementia compared to people without dementia across care settings, particularly in people with severe cognitive impairment. ${ }^{40-42}$

\section{Canada}

In Canada, a recent report on opioid use in nursing home patients describes lower prescribing rates compared to the USA, with a contrasting trend towards increased use among people with and without dementia. ${ }^{43}$ In Ontario, Iaboni et al (2019) found that overall prescription of opioids to longterm care residents increased from $15.8 \%$ in $2009 / 10$ to $19.6 \%$ in $2016 / 17(<0.001)$; in people without dementia a $21.8 \%$ increase, from $21.3 \%$ to $26.0 \%$, was reported $(<0.001)$, while people with dementia had a reported $38.5 \%$ increase, from $11.8 \%$ to $16.3 \%(\mathrm{p}<0.001)$, indicating that they are still significantly less likely to receive opioid treatment compared to people without dementia. ${ }^{43}$

\section{Nordic Countries}

In the Nordic countries, multiple studies over the last 5 years have reported few differences in opioid prescribing rates between people with and without dementia. Studies in nursing homes in Norway and Denmark have reported that elderly people with dementia are less likely to receive opioids than people without dementia, but this difference appears to be mainly driven by less use of weak opioids in nursing home residents with dementia. ${ }^{44,45}$ The most recent estimate from Norway, based on data from a 2011 cohort, reported opioid use in $23.8 \%$ of nursing home patients and found that having dementia was not associated with a significant difference in the likelihood of receiving a strong opioid. ${ }^{44}$ In a study involving the entire population of Denmark in 2010, 37.8\% of nursing home residents with dementia received any opioid compared to $43.0 \%$ of those without dementia $(\mathrm{p}<0.05)$. However, there was no significant difference for strong opioids $(27.8 \%$ vs $28.1 \%, \mathrm{p}>0.05) .{ }^{44}$ The same study found that people with dementia in the community-dwelling population were more likely to receive opioid analgesics compared to those without dementia (27.5\% vs $16.9 \%$, adjusted OR $1.27,95 \%$ CI $1.22-1.31) .{ }^{44}$ Updated Danish registry data (2017) showed that use of opioids was still significantly higher in elderly people with dementia compared to elderly without dementia, although this study did not adjust for living status. ${ }^{46}$

In Finland, Hamina et al (2017) investigated opioid use among all community-dwelling people diagnosed with Alzheimer's disease (AD) compared to matched controls 
from 2005 to $2011(62,074$ persons with and 62,074 persons without AD). ${ }^{47}$ They found that although long-term opioid use was more common among persons without $\mathrm{AD}$ (8.7\% vs $7.2 \%, \mathrm{P}<0.001$ ), among opioid users with $\mathrm{AD}$ a higher proportion were long-term users $(34.2 \%$ vs $32.3 \%, \mathrm{P}<0.001) .{ }^{47}$ Moreover, long-term use of transdermal opioids was more than twice as prevalent among opioid users with $\mathrm{AD}(13.2 \%$ vs $5.5 \%, \mathrm{p}<0.0001) .{ }^{47}$ Roitto et al (2019) investigated 14-year trends (2003-2017) in opioid use among institutionalized older people in Helsinki. ${ }^{48}$ Over this period, opioid use increased significantly amongst people with and without dementia, living in both nursing homes and assisted living facilities. In nursing homes and assisted living facilities, fewer people with dementia used opioids compared to those without dementia, and opioid use in nursing homes increased more rapidly among residents with dementia compared to those without dementia $(\mathrm{p}<0.01){ }^{48}$

\section{Regional Differences}

Data from the 2010 population study in Denmark have also been used to estimate municipal and regional differences in opioid prescribing. Interestingly, differences between municipalities were most pronounced among home-dwelling elderly people with dementia, with a 4 -fold difference observed (ranging from $9.4 \%$ to $36.8 \%$ ) compared to a 1.6 -fold difference among homedwelling elderly people without dementia (ranging from $12.7 \%$ to $20.2 \%){ }^{49}$ Among nursing home patients an equal and approximately 2 -fold difference was observed, ranging from $26.5 \%$ to $55.2 \%$ for people with dementia and from $31.8 \%$ to $60.4 \%$ for nursing home patients not diagnosed with dementia. ${ }^{49}$ As the largest variations were found between municipalities rather than between larger regions, the authors suggested that primary care practice, which is organized at the municipal level, is the likely explanation for inconsistencies. No comparable study has been conducted in any other country. Nevertheless, given the large variations in prescribing practice between countries as outlined in this review, it is clear that there is a lack of coherent clinical guidelines for the assessment and treatment of pain in people with dementia, and/or a lack of implementation of such guidelines in clinical practice. Both prescribing traditions and practices within national medical communities, and differences between individual clinicians, likely contribute to the large observed variations in opioid treatment.

\section{Undertreatment or Overtreatment?}

A further important issue is whether countries with high opioid prescription rates actually achieve optimal treatment of pain. To exemplify, a study of 327 nursing home patients with dementia and behavioral disturbances in Norway found that of the patients' prescribed opioids (19.3\% of the total), $79.4 \%$ were still in pain. ${ }^{48}$ Practice differences in the management of moderate-to-severe (chronic) pain are illustrated by studies that compared the post-operative treatment of pain (after fracture) in people with and without dementia. In Denmark, JensenDahm et al (2016) investigated the postoperative treatment of pain during the first 72 hours after hip fracture among all elderly patients treated in the Copenhagen University Hospital region in 2009. ${ }^{50}$ The study found that although people with dementia were equally likely to receive paracetamol and opioids, they received slightly lower dosages and more often received the medication pro re nata or "as needed". Analyses were controlled for living status, which did not impact the results. In Finland, Lintula et al (2020) investigated the prevalence of opioid use in communitydwelling persons (2342 with AD and 1615 without AD) with hip fracture during the first 6 months after discharge from the hospital. ${ }^{51}$ The study found that opioid use was significantly more common among people with AD compared to those without $\mathrm{AD}\left(39.5 \%\right.$ vs $31.2 \%$, $\chi^{2}$ test, $\mathrm{p}<0.0001$ ), and that people with $\mathrm{AD}$ were more likely to use strong opioids during the first 3 months after discharge. Buprenorphine use was more frequent in people with $\mathrm{AD}$ during the entire follow-up period, while people with $\mathrm{AD}$ used weak opioids less frequently during the first month after discharge compared to those without $\mathrm{AD}$. In the USA, Mehta et al (2020) investigated opioid use among long-term care residents during a year in which a fracture was diagnosed during an emergency department visit, and found that within 7 days of discharge, $53.3 \%$ of people without dementia used an opioid, while corresponding figures for people with mild, moderate, and severe dementia were $47.5 \%, 43.6 \%$, and $35.7 \%$, respectively. ${ }^{40}$ In Australia, Inacio et al (2016) found that having dementia was a risk factor for new chronic opioid use following total hip arthroplasty $(\mathrm{OR}=2.19,95 \% \mathrm{CI}$ 1.06 to 6.39$).{ }^{51}$

This review has highlighted trends and developments in opioid treatment for elderly people with dementia over the last 5 years. We found that there is still considerable geographic variation in the use of opioids, both between 
and within countries, and wider variation among people with dementia compared to those without dementia. Rates of reported opioid use among nursing home patients with dementia ranged from less than $3 \%$ in Poland to $55 \%$ in Denmark. $^{38,49}$ Furthermore, several studies reported a gradient of decreasing opioid use with more severe cognitive impairment. ${ }^{40,41}$ While the ideal level of opioid treatment in people with dementia has yet to be determined, current evidence shows that undertreatment of pain in people with dementia is still an important clinical issue, although the extent of the problem varies with prescribing practice both between countries and clinical settings. It is important to note that greater use of opioids is not equivalent to appropriate use - several studies have reported that people with dementia are more likely to continue strong opioid treatment long term, and that transdermal buprenorphine is strongly associated with chronic opioid use. $^{47,51,52}$ This may indicate insufficient reevaluation of appropriate opioid treatment in people with dementia and might suggest that a vulnerable group is at risk of drug-related harm. ${ }^{53}$

\section{Non-Pharmacological Treatments}

Given the fact that older persons have more comorbidity and less organ capacity, they are more prone to both polypharmacy and the negative effects of polypharmacy. ${ }^{54}$ In addition, people with dementia are not only more susceptible to side-effects because of their age but also due to their brain disease which makes them more vulnerable to delirium. ${ }^{55}$ Therefore, when they are effective, non-pharmacological interventions should be the first choice. Regarding behavioral problems, many non-pharmacological interventions have been shown to be effective in people with dementia, ${ }^{56}$ although implementation in practice presents challenges. ${ }^{57}$ Nonpharmacological interventions for pain have been reported, but evidence concerning people with dementia is unfortunately scarce. ${ }^{20} \mathrm{~A}$ recent study in a care home setting showed efficacy for massage and exercise on both pain and as-needed medication. ${ }^{58}$ Interestingly, the effects were stronger in the half of the sample with dementia. Another study found evidence for the effectiveness of massage and human interaction, but not for aromatherapy. ${ }^{59}$ Also, a relatively small study found significant effects on pain of the PARO robot seal in a population with dementia. ${ }^{60}$

Interestingly, interventions effective for behavioral problems in dementia are also commonly effective on pain, and vice versa. ${ }^{61}$ Nowadays, complex behavioral problems are believed to be best handled by stepwise, multi-aspect programs, with a large non-pharmacological component. ${ }^{62}$ Some have also shown a beneficial effect on pain. ${ }^{63}$ Over the next decade, we hope to see a greater emphasis on high-quality studies evaluating the efficacy and feasibility of non-pharmacological interventions.

\section{Conclusion}

This update of recent literature supports continuing concerns regarding the proper management of pain in persons with dementia. Clinicians seem to still have difficulties evaluating the expression and intensity of pain, and difficulties monitoring the effects of analgesic medication, in persons with dementia. Literature suggests that both starting and stopping opioid use is complicated, as reflected in the long-term use of opioid patches reported in several studies. ${ }^{64}$ Therefore, better use should be made of our current understanding of the mechanisms of pain expression, and available clinical observational assessment instruments could be better implemented. The use of more elaborate technical pain-sensing systems is promising but unfortunately still remains in the realms of science fiction. Non-pharmacological interventions should be more promoted as management or co-management options both in research and clinical practice.

\section{Disclosure}

None of the authors have a conflict of interest to disclose.

\section{References}

1. Stelzmann RA, Norman Schnitzlein H, Reed Murtagh F. An English translation of alzheimer's 1907 paper, "über eine eigenartige erkankung der hirnrinde". Clin Anatomy. 1995;8(6):429-431. doi:10.1002/ ca.980080612

2. Taheri-Targhi S, Gjedde A, Araj-Khodaei M, et al. Avicenna (980-1037 CE) and his early description and classification of dementia. J Alzheimers Dis. 2019;71(4):1093-1098.

3. Rizzi L, Rosset I, Roriz-Cruz M. Global epidemiology of dementia: alzheimer's and vascular types. Biomed Res Int. 2014;2014:908915. doi:10.1155/2014/908915

4. Melis RJF, Haaksma ML, Muniz-Terrera G. Understanding and predicting the longitudinal course of dementia. Curr Opin Psychiatry. 2019;32(2):123-129. doi:10.1097/YCO.0000000000000482

5. Cotter VT. The burden of dementia. Am J Manag Care. 2007;13(Suppl 8):S193-197.

6. Husebo BS, Achterberg W, Flo E. Identifying and managing pain in people with alzheimer's disease and other types of dementia: a systematic review. CNS Drugs. 2016;30(6):481-497. doi:10.1007/ s40263-016-0342-7

7. van der Steen JT, Lennaerts H, Hommel D, et al. Dementia and Parkinson's disease: similar and divergent challenges in providing palliative care. Front Neurol. 2019;10:54. doi:10.3389/fneur.2019.00054 
8. Scherder EJ, Sergeant JA, Swaab DF. Pain processing in dementia and its relation to neuropathology. Lancet Neurol. 2003;2 (11):677-686. doi:10.1016/S1474-4422(03)00556-8

9. Sewards TV, Sewards MA. The medial pain system: neural representations of the motivational aspect of pain. Brain Res Bull. 2002;59 (3): $163-180$.

10. Monroe TB, Gore JC, Chen LM, Mion LC, Cowan RL. Pain in people with Alzheimer disease: potential applications for psychophysical and neurophysiological research. $J$ Geriatr Psychiatry Neurol. 2012;25(4):240-255. doi:10.1177/0891988712466457

11. Achterberg WP, Pieper MJ, van Dalen-kok AH, et al. Pain management in patients with dementia. Clin Interv Aging. 2013;8:1471-1482. doi:10.2147/CIA.S36739

12. Defrin R, Amanzio M, de Tommaso M, et al. Experimental pain processing in individuals with cognitive impairment: current state of the science. Pain. 2015;156(8):1396-1408. doi:10.1097/j. pain. 0000000000000195

13. Kunz M, Scharmann S, Hemmeter U, Schepelmann K, Lautenbacher S. The facial expression of pain in patients with dementia. Pain. 2007;133 (1-3):221-228. doi:10.1016/j.pain.2007.09.007

14. Beach PA, Huck JT, Miranda MM, Foley KT, Bozoki AC. Effects of Alzheimer disease on the facial expression of pain. Clin J Pain. 2016;32(6):478-487. doi:10.1097/AJP.0000000000000302

15. Ekman PEFW. Facial Action Coding System. Palo Alto, CA: Consulting Psychologists Press; 1978.

16. Kunz M, Meixner D, Lautenbacher S. Facial muscle movements encoding pain-a systematic review. Pain. 2019;160(3):535-549. doi:10.1097/j.pain.0000000000001424

17. Kunz M, Lautenbacher S. The faces of pain: a cluster analysis of individual differences in facial activity patterns of pain. Eur J Pain. 2014;18(6):813-823. doi:10.1002/j.15322149.2013.00421.x

18. Kunz M, Prkachin K, Solomon PE, Lautenbacher S. Faces of clinical pain: inter-individual facial activity patterns in shoulder pain patients. Eur J Pain. 2020. doi:10.1002/ejp.1691

19. Herr K, Coyne PJ, Ely E, Gelinas C, Manworren RCB. Pain assessment in the patient unable to self-report: clinical practice recommendations in support of the ASPMN 2019 position statement. Pain Manag Nurs. 2019;20(5):404-417. doi:10.1016/j.pmn.2019.07.005

20. Achterberg W, Lautenbacher S, Husebo B, Erdal A, Herr K. Pain in dementia. Pain Rep. 2020;5(1):e803. doi:10.1097/ PR9.0000000000000803

21. Zwakhalen SM, Hamers JP, Abu-Saad HH, Berger MP. Pain in elderly people with severe dementia: a systematic review of behavioural pain assessment tools. BMC Geriatr. 2006;6:3.

22. Jordan A, Hughes J, Pakresi M, Hepburn S, O'Brien JT. The utility of PAINAD in assessing pain in a UK population with severe dementia. Int $J$ Geriatr Psychiatry. 2011;26(2):118-126. doi:10.1002/gps.2489

23. Warden V, Hurley AC, Volicer L. Development and psychometric evaluation of the Pain Assessment in Advanced Dementia (PAINAD) scale. J Am Med Dir Assoc. 2003;4(1):9-15. doi:10.1097/01. JAM.0000043422.31640.F7

24. Fuchs-Lacelle S, Hadjistavropoulos T. Development and preliminary validation of the pain assessment checklist for seniors with limited ability to communicate (PACSLAC). Pain Manag Nurs. 2004;5 (1):37-49. doi:10.1016/j.pmn.2003.10.001

25. Wary B, Doloplus C. [Doloplus-2, a scale for pain measurement]. Soins Gerontol. 1999;(19):25-27.

26. Holen JC, Saltvedt I, Fayers PM, Hjermstad MJ, Loge JH, Kaasa S. Doloplus-2, a valid tool for behavioural pain assessment? BMC Geriatr. 2007;7:29. doi:10.1186/1471-2318-7-29

27. Ersek M, Neradilek MB, Herr K, et al. Psychometric evaluation of a pain intensity measure for persons with Dementia. Pain Med. 2019;20(6):1093-1104. doi:10.1093/pm/pny166
28. Kunz M, de Waal MWM, Achterberg WP, et al. The Pain Assessment in Impaired Cognition scale (PAIC15): a multidisciplinary and international approach to develop and test a meta-tool for pain assessment in impaired cognition, especially dementia. Eur J Pain. 2020;24 (1):192-208. doi:10.1002/ejp.1477

29. de Waal MWM, van Dalen-kok AH, de Vet HCW, et al. Observational pain assessment in older persons with dementia in four countries: observer agreement of items and factor structure of the pain assessment in impaired cognition. Eur J Pain. 2020;24 (2):279-296. doi:10.1002/ejp.1484

30. Kunz M, Seuss D, Hassan T, et al. Problems of video-based pain detection in patients with dementia: a road map to an interdisciplinary solution. BMC Geriatr. 2017;17(1):33. doi:10.1186/s12877-017-0427-2

31. Hassan T, Seus D, Wollenberg J, et al. Automatic detection of pain from facial expressions: a survey. IEEE Trans Pattern Anal Mach Intell. 2019. doi:10.1109/TPAMI.2019.2958341

32. Scherder EJ, Bouma A. Is decreased use of analgesics in Alzheimer disease due to a change in the affective component of pain? Alzheimer Dis Assoc Disord. 1997;11(3):171-174. doi:10.1097/ 00002093-199709000-00010

33. Mantyselka P, Hartikainen S, Louhivuori-Laako K, Sulkava R. Effects of dementia on perceived daily pain in home-dwelling elderly people: a population-based study. Age Ageing. 2004;33(5):496-499. doi:10.1093/ageing/afh 165

34. Nygaard HA, Jarland M. Are nursing home patients with dementia diagnosis at increased risk for inadequate pain treatment? Int J Geriatr Psychiatry. 2005;20(8):730-737. doi:10.1002/gps.1350

35. Cornali C, Franzoni S, Gatti S, Trabucchi M. Diagnosis of chronic pain caused by osteoarthritis and prescription of analgesics in patients with cognitive impairment. J Am Med Dir Assoc. 2006;7(1):1-5. doi:10.1016/j.jamda.2005.06.010

36. Corbett A, Husebo BS, Achterberg WP, Aarsland D, Erdal A, Flo E. The importance of pain management in older people with dementia. Br Med Bull. 2014;111(1):139-148. doi:10.1093/bmb/ldu023

37. Eccleston C, Fisher E, Thomas KH, et al. Interventions for the reduction of prescribed opioid use in chronic non-cancer pain. Cochrane Database Syst Rev. 2017;11(11):Cd010323. doi:10.1002/ 14651858.CD010323.pub3

38. Neumann-Podczaska A, Nowak T, Suwalska A, et al. Analgesic use among nursing homes residents, with and without dementia, in Poland. Clin Interv Aging. 2016;11:335-340. doi:10.2147/CIA.S101475

39. Marengoni A, Nobili A, Corli O, et al. The stigma of low opioid prescription in the hospitalized multimorbid elderly in Italy. Intern Emerg Med. 2015;10(3):305-313. doi:10.1007/s11739-014-1131-2

40. Mehta HB, Kuo YF, Raji M, Li S, Westra J, Goodwin JS. Time trends in opioid use by dementia severity in long-term care nursing home residents. J Am Med Dir Assoc. 2020;22:124-131.e1. doi:10.1016/j. jamda.2020.04.029

41. Hunnicutt JN, Chrysanthopoulou SA, Ulbricht CM, Hume AL, Tjia J, Lapane KL. Prevalence of long-term opioid use in long-stay nursing home residents. J Am Geriatr Soc. 2018;66(1):48-55. doi:10.1111/ jgs. 15080

42. Shen C, Zhao X, Dwibedi N, Wiener RC, Findley PA, Sambamoorthi U. Opioid use and the presence of Alzheimer's disease and related dementias among elderly Medicare beneficiaries diagnosed with chronic pain conditions. Alzheimers Dement. 2018;4:661-668. doi:10.1016/j.trci.2018.10.012

43. Iaboni A, Campitelli MA, Bronskill SE, et al. Time trends in opioid prescribing among Ontario long-term care residents: a repeated cross-sectional study. CMAJ Open. 2019;7(3):E582-E589. doi:10.9778/cmajo.20190052

44. Jensen-Dahm C, Gasse C, Astrup A, Mortensen PB, Waldemar G. Frequent use of opioids in patients with dementia and nursing home residents: a study of the entire elderly population of Denmark. Alzheimers Dement. 2015;11(6):691-699. doi:10.1016/j. jalz.2014.06.013 
45. Sandvik R, Selbaek G, Kirkevold O, Aarsland D, Husebo BS Analgesic prescribing patterns in Norwegian nursing homes from 2000 to 2011: trend analyses of four data samples. Age Ageing. 2016;45(1):54-60. doi:10.1093/ageing/afv184

46. Sorensen AMS, Tarp S, Johannsen P, et al. Analgesics use and withdrawal in people with dementia - a register-based Danish study and a systematic review. Dan Med J. 2019;66:12.

47. Hamina A, Taipale H, Tanskanen A, et al. Long-term use of opioids for nonmalignant pain among community-dwelling persons with and without Alzheimer disease in Finland: a nationwide register-based study. Pain. 2017;158:2. doi:10.1097/j.pain.0000000000000752

48. Roitto HM, Kautiainen H, Aalto UL, Öhman H, Laurila J, Pitkälä KH. Fourteen-year trends in the use of psychotropic medications, opioids, and other sedatives among institutionalized older people in Helsinki, Finland. J Am Med Dir Assoc. 2019;20(3):305-311. doi:10.1016/j.jamda.2018.12.022

49. Jensen-Dahm C, Zakarias JK, Gasse C, Waldemar G. Geographical variation in opioid use in elderly patients with dementia: a nationwide study. J Alzheimers Dis. 2019;70(4):1209-1216. doi:10.3233/JAD-190413

50. Jensen-Dahm C, Palm H, Gasse C, Dahl JB, Waldemar G. Postoperative treatment of pain after hip fracture in elderly patients with Dementia. Dement Geriatr Cogn Disord. 2016;41(3-4):181-191. doi:10.1159/000444704

51. Lintula E, Tiihonen M, Taipale H, et al. Opioid use after hospital care due to hip fracture among community-dwelling persons with and without Alzheimer's Disease. Drugs Aging. 2020;37(3):193-203. doi:10.1007/s40266-019-00734-0

52. Inacio MCS, Hansen C, Pratt NL, Graves SE, Roughead EE. Risk factors for persistent and new chronic opioid use in patients undergoing total hip arthroplasty: a retrospective cohort study. BMJ Open. 2016;6(4):e010664. doi:10.1136/bmjopen-2015-010664

53. Erdal A, Flo E, Aarsland D, et al. Tolerability of buprenorphine transdermal system in nursing home patients with advanced dementia: a randomized, placebo-controlled trial (DEP.PAIN.DEM). Clin Interv Aging. 2018;13:935-946. doi:10.2147/CIA.S161052

54. Gareri P, Cotroneo AM, Pontieri MT, Palleria C, De Sarro G. The risk of polypharmacy and potentially inappropriate drugs in residential care dementia patients: tips from the PharE study. Aging Clin Exp Res. 2020. doi:10.1007/s40520-020-01719-5

55. Zuliani G, Bonetti F, Magon S, et al. Subsyndromal delirium and its determinants in elderly patients hospitalized for acute medical illness. J Gerontol Biol Sci Med Sci. 2013;68(10):1296-1302. doi:10.1093/ gerona/glt021
56. Dyer SM, Harrison SL, Laver K, Whitehead C, Crotty M. An overview of systematic reviews of pharmacological and non-pharmacological interventions for the treatment of behavioral and psychological symptoms of dementia. Int Psychogeriatr. 2018;30(3):295-309. doi:10.1017/S1041610217002344

57. Cohen-Mansfield J, Thein K, Marx MS, Dakheel-Ali M. What are the barriers to performing nonpharmacological interventions for behavioral symptoms in the nursing home? $\mathrm{J}$ Am Med Dir Assoc. 2012;13(4):400-405. doi:10.1016/j.jamda.2011.07.006

58. Ellis JM, Wells Y, Ong JSM. Non-pharmacological approaches to pain management in residential aged care: a pre-post-Test study. Clin Gerontol. 2019;42(3):286-296. doi:10.1080/07317115.2017.1399189

59. Anderson AR, Deng J, Anthony RS, Atalla SA, Monroe TB. Using complementary and alternative medicine to treat pain and agitation in dementia: a review of randomized controlled trials from long-term care with potential use in critical care. Crit Care Nurs Clin North Am. 2017;29(4):519-537. doi:10.1016/j.cnc.2017.08.010

60. Pu L, Moyle W, Jones C, Todorovic M. The effect of using PARO for people living with dementia and chronic pain: a pilot randomized controlled trial. J Am Med Dir Assoc. 2020;21(8):1079-1085.

61. Pieper MJ, van Dalen-kok AH, Francke AL, et al. Interventions targeting pain or behaviour in dementia: a systematic review. Ageing Res Rev. 2013;12(4):1042-1055. doi:10.1016/j.arr.2013.05.002

62. Pieper MJ, Francke AL, van der Steen JT, et al. Effects of a stepwise multidisciplinary intervention for challenging behavior in advanced dementia: a cluster randomized controlled trial. J Am Geriatr Soc. 2016;64(2):261-269.

63. Pieper MJC, van der Steen JT, Francke AL, Scherder EJA, Twisk JWR, Achterberg WP. Effects on pain of a stepwise multidisciplinary intervention (STA OP!) that targets pain and behavior in advanced dementia: a cluster randomized controlled trial. Palliat Med. 2018;32(3):682-692. doi:10.1177/0269216316689237

64. Achterberg W, Lautenbacher S. Editorial: pain in Dementia: a distressing combination of several factors. Curr Alzheimer Res. 2017;14(5):468-470. doi:10.2174/156720501405170403224615
Journal of Pain Research

\section{Publish your work in this journal}

The Journal of Pain Research is an international, peer reviewed, open access, online journal that welcomes laboratory and clinical findings in the fields of pain research and the prevention and management of pain. Original research, reviews, symposium reports, hypothesis formation and commentaries are all considered for publication. The manuscript

Submit your manuscript here: https://www.dovepress.com/journal-of-pain-research-journa management system is completely online and includes a very quick and fair peer-review system, which is all easy to use. Visit http:// www.dovepress.com/testimonials.php to read real quotes from published authors. 